\title{
Super-resolution PIV using multi-frame displacement information
}

\author{
Zhenxing Ouyang ${ }^{1}$, Hua Yang ${ }^{1 *}$, Yunkang Cao ${ }^{1}$, Zhen Yang ${ }^{1}$, Zhouping Yin \\ ${ }^{1}$ State Key Laboratory of Digital Manufacturing Equipment and Technology, Huazhong University of \\ Science and Technology, Wuhan, 430074, China \\ * huayang@ hust.edu.cn
}

\begin{abstract}
High-resolution (HR) fluid-flow velocity information is important to reliably analyze fluid measurements in particle image velocimetry (PIV), such as the boundary layer and turbulent flow. Efforts in PIV to enhance the resolution of flow fields are mainly based on single-frame information, which follows the velocity field estimation and may influence the final reconstruction accuracy. In this study, we propose a novel super-resolution (SR) reconstruction technology from another perspective, which consists of two parts: a multi-frame imaging system and a Bayesian-based multi-frame SR reconstruction algorithm. First, a splitbased imaging system is developed to obtain particle image pairs with fixed displacements. Subsequently, we present a Bayesian-based multi-frame SR (BMFSR) reconstruction algorithm to obtain an SR particle image. Multi-frame particle images collected by the developed system are used as the input low-resolution images for the following novel SR reconstruction algorithm. Synthetic and experimental particle images have been tested to verify the performance of the proposed technology, and the results are compared with the traditional and advanced reconstruction methods in PIV. The results and comparisons show that the proposed technology successfully achieves good performance in obtaining finer particle images and a more accurate velocity field.
\end{abstract}

\section{Introduction}

High-resolution (HR) fluid-flow velocity information is important to reliably analyze fluid measurements in particle image velocimetry (PIV), such as the boundary layer and turbulent flow. However, fluid-flow data are often sparse, have limited spatial resolution and are noisy in real life for various reasons, such as measuring device imperfections or instability in the observed scene, sensor resolution limitations in experiments or insufficient mesh size in computational fluid dynamics (CFD) simulations (Gao et al., 2020). Therefore, it is necessary to improve the flow field resolution to explore many different boundaries, small-scale structures, turbulence transitions and so on. In PIV measurement, the HR flow field can be obtained by CFD methods, such as large eddy simulation (LES) and direct numerical simulation (DNS) (Liu et al., 2020). Nevertheless, simulations can only model the flow ideally and cannot fully analyze the real situation. Consequently, it is essential and practically useful to reconstruct flow information from spatiotemporal low-resolution (LR) data.

Super-resolution (SR) reconstruction is a classic technique for improving the resolution of an imaging system. It can realize the resolution enhancement of a larger imaging system with HR data on a smaller domain (Brunton et al., 2020). For decades, various SR reconstruction methods have been proposed, which can be divided into two types: traditional methods and deep learning (DL) methods. Traditional SR reconstruction methods mainly utilize interpolation methods to improve the flow field resolution. Keys (1981) employed bicubic interpolation based on the filter operation with low-pass characteristics to increase the resolution of digital images. Takehara et al. (2000) proposed an SR reconstruction method based on a Kalman filter and $\chi^{2}$-testing and verified its performance on two synthetic datasets. Next, efforts (Gunes and Rist, 2007; Alfonsi et al., 2013; He and Liu, 2017) that applied proper orthogonal decomposition (POD) modes extracted from the DNS dataset to obtain the corresponding coefficients and reconstruct the HR flow field were made. These traditional SR reconstruction methods are based on the least-squares approximation to estimate the weighting factors between the LR data and HR data, which limits spatial resolution improvement to some extent. 
In the last two decades, the application of SR reconstruction technology based on DL in fluid dynamics has attracted increasing attention. DL reconstruction is a data-driven technology that can provide an effective method for generating HR flow fields quickly without iteratively solving partial differential equations (PDEs). Fukami et al. (2019a,b) adopted the convolutional neural network (CNN) and hybrid downsampled skip-connection multiscale models to reconstruct two-dimensional attenuated isotropic turbulence. (Deng et al., 2019) proposed a network-based generative adversarial network-based artificial intelligence framework to enhance the spatial resolution of complicated wake flow behind two side-by-side cylinders; however, (Liu et al., 2020) proposed a multitime path CNN. To reduce the increase in the visual complexity to an LR input, which is caused by data-driven upsampling approaches, various efforts (Raissi et al., 2019; Gao et al., 2020; Dwivedi et al., 2021) have been proposed. Despite the advantages, the success of these DL models mainly relies on a large quantity of offline HR data as labels.

All the models mentioned above, whether traditional or deep learning-based SR reconstruction methods, only utilize the spatial information on the LR fluid field based on single-frame data and reconstruct following the estimation of the velocity field. However, the information provided by only a two successive images is limited. Moreover, the LR data that are used to generate the weighting parameters between the LR data and HR data are not obtained simultaneously, resulting in the pertinence of these LR data being low. Furthermore, the LR data used in the methods mentioned before are generally not the image itself, which may influence reconstruction accuracy. Thus, in this study, a novel SR reconstruction technology is presented from another perspective: SR particle image reconstruction using multi-frame displacement information. It is proposed based on the idea of simultaneously collecting two LR particle images with a fixed displacement and then generating the corresponding SR particle image with a novel SR reconstruction algorithm. As a result, this novel reconstruction technology consists of two parts: a multi-frame imaging system and a Bayesian-based multi-frame SR (BMFSR) reconstruction algorithm. First, a split-based imaging system is developed to obtain pairs of particle images with fixed displacements. Subsequently, an algorithm named BMFSR is proposed to obtain an SR particle image. Multi-frame particle images collected by the developed system are used as the input LR images for the following novel SR reconstruction algorithm. In this way, more correlated particle images can be collected simultaneously, and more LR spatial information can be used. Finally, we determine whether the novel technology can reconstruct better results.

The rest of this paper is structured as follows: Sect. 2 introduces the basic scheme of the novel SR technology, including the multi-frame imaging system and the Bayesian-based multi-frame SR reconstruction algorithm. Subsequently, experimental evaluations on synthetic images and real data are demonstrated in Sect. 3. Finally, conclusions are discussed in Sect. 4.

\section{The proposed super-resolution reconstruction technology}

The proposed super-resolution reconstruction technology is a novel SR particle image reconstruction method using multi-frame displacement information. This technology contains two main parts: a multi-frame imaging system to collect double LR particle images simultaneously with a fixed displacement and a Bayesianbased multi-frame SR (BMFSR) reconstruction algorithm to generate the corresponding HR particle image. Finally, the HR velocity field can be obtained with the corresponding two successive reconstructed HR particle images with an interval time.

\subsection{Multi-frame imaging system}

As mentioned above, traditional and deep learning SR reconstruction methods generate HR flow fields based on LR single-frame data. Additionally, the LR data used are obtained from a series of two successive particle frames. Different pairs of two successive frames are collected at different times, resulting in limited spatial correlation between them, which limits the spatial information and may influence the final reconstruction accuracy. Hence, in this study, we design a multi-frame imaging system to collect two particle images simultaneously with a fixed displacement time to improve spatial relativity. The schematic and photographs of this system are shown in Fig. 1.

As shown in Fig. 1 (a), this multi-frame imaging system consists of three parts: a spectral region, control unit and transmit unit. The particle image intensity is defined as $I$ and the collecting time is $t$. To obtain the two relative particle images $I_{t}^{1}$ and $I_{t}^{2}$ at the same time $t$ with a fixed displacement, in the spectral region, a splitter is designed to split the incident light into two identical parts collected by two high-rate sensors, sensor 1 and sensor 2, which are fixed with a preset constant displacement, and the displacement is generated with a contact translation stage (shown in Fig. 1 (c)). The particle image collection is controlled 
by the control unit, which mainly consists of three parts, namely, a field programmable gate array (FPGA) controlling the exposure of the two sensors, double data rate RAM (DDR) buffering the image data and a solid-state disk (SSD) storing all particle images collected within a certain time. After collection, particle images $I_{t}^{1}$ and $I_{t}^{2}$ are transmitted to a personal computer through the transmit unit to be estimated with the proposed BMFSR reconstruction algorithm. The outer and inner photographs of this multi-frame imaging system are shown in Fig. 1 (b) and (c), respectively.
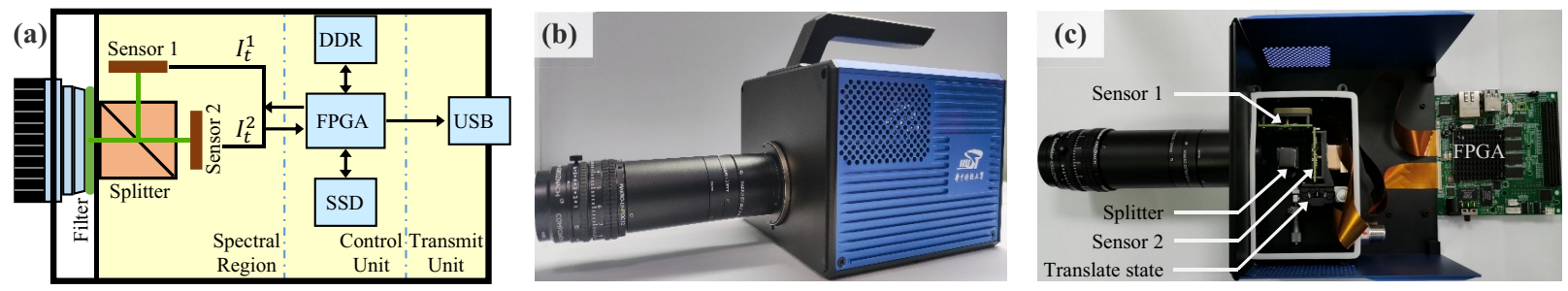

Figure 1: Schematic (a), outer photograph (b) and inner photograph (c) of the multi-frame imaging system.

\subsection{Bayesian-based multi-frame SR reconstruction algorithm}

Current SR reconstruction methods in PIV measurement are mainly based on a single frame, that is, one input and one output. The formulation of the single-frame-based SR reconstruction methods can be expressed as:

$$
\underset{\psi^{H R}}{\arg \min }\left\|\psi^{L R}-\mathbf{S K} \psi^{H R}\right\|^{2}+\eta R\left(\psi^{H R}\right)
$$

where $\psi^{L R}$ denotes the LR flow data, such as the velocity field, and $\psi^{H R}$ is the corresponding HR data to be reconstructed. $\mathbf{S}$ and $\mathbf{K}$ correspond to downsampling and filtering with a blur kernel, respectively. $R$ is a function of the HR data $\psi^{H R}$ to constrain the first term so that the solution is unique and the parameter $\eta$ balances the first and second terms. With increasing input LR image numbers, the ideal HR flow data can be obtained from Eq. 1.

However, LR particle images utilized in the single-frame-based methods are collected at different times, whether within one successive two-frame pair to estimate one LR velocity field or without, resulting in the spatial information of a single-frame particle image being limited, which may influence the final reconstruction accuracy. Hence, based on the multi-frame imaging system, we also propose a Bayesian-based multi-frame SR (BMFSR) reconstruction method, which involves two particle frames collected at the same time with a constant displacement into the reconstruction function and is modified from Liu and Sun (2014). This novel SR algorithm is proposed from another perspective: SR particle image reconstruction. Let the image intensity be $I$, the first collecting time be $t$ and the second be $t+\Delta t$, where $\Delta t$ is the interval time. Then, taking time $t$ as an example, the new reconstruction formulation is as follows:

$$
J_{t}^{*}=\underset{J_{t}}{\arg \min } \theta_{1}\left\|\mathbf{S K} J_{t}-I_{t}^{1}\right\|+\eta\left\|\nabla J_{t}\right\|+\theta_{2}\left\|\mathbf{S K K} F_{\mathbf{w}} J_{t}-I_{t}^{2}\right\|
$$

where $J_{t}{ }^{*}$ is the object function to be minimized and $J_{t}$ is the upsampling image to be reconstructed at time $t$. $I_{t}^{1}$ and $I_{t}^{2}$ are the LR particle images collected by sensor 1 and sensor 2 at time $t . \nabla J_{t}$ is the gradient of HR image $J_{t} . F_{\mathbf{w}}$ is the warping matrix corresponding to the displacements $\mathbf{w}$ between $J_{t}^{1}$ and $J_{t}^{2}$. $\eta$ is a parameter that balances the influence of sparsity on the derivative filter to model the priors of HR image $J_{t}$ and is usually set to 0.02. $\theta_{1}$ and $\theta_{2}$ are parameters that control the noise and outlier when $J_{t}$ is warped to generate the adjacent frame. Note that L1-norm penalization is adopted instead of L2-norm used in Eq. 1, because that L1-norm can achieve better stability and convergence (Seong et al., 2019).

By solving Eq. 2, the HR image $I_{t}^{H R}$ can be obtained. In the same way, the correlated HR image $I_{t+\Delta t}^{H R}$ will be obtained with Eq. 2 from the other two frames $I_{t+\Delta t}^{1}$ and $I_{t+\Delta t}^{2}$. Finally, the velocity field of the flow is estimated with an open source (Vejrazka, 2021), which is a cross-correlation algorithm, named DCC in this paper. Bicubic interpolation is used to obtain a full-size velocity field.

With the Bayesian-based multi-frame SR reconstruction algorithm, more spatial information at one time can be used to reconstruct the HR data, resulting in the reconstruction accuracy improvement. 


\subsection{Minimization and parameter setting}

For the novel SR reconstruction algorithm, we can estimate the HR image by solving Eq. 2 when the current flow field $\mathbf{w}$, blur kernel $\mathbf{K}$ and noise levels $\theta_{1}$ and $\theta_{2}$ are given. To simplify the estimation, we replace the L1-norm with a non-quadratic penalty function $\phi\left(s^{2}\right)=\sqrt{s^{2}+\tau^{2}}$. The symbol $\tau$ is a prefixed small constant, which is usually set as $\tau=0.01$, to ensure that $\phi$ is strictly convex.

This object function of Eq. 2 can be solved by the iterated reweighted least squares (IRLS) method (Liu et al., 2009), which iteratively solves the following linear system:

$$
\left[\theta_{1} \mathbf{K}^{T} \mathbf{S}^{T} P_{1} \mathbf{S K}+\eta\left(\left(J_{t}\right)_{x}^{T} P_{S}\left(J_{t}\right)_{x}+\left(J_{t}\right)_{y}^{T} P_{S}\left(J_{t}\right)_{y}\right)+\theta_{2} F_{\mathbf{w}}^{T} \mathbf{K}^{T} \mathbf{S}^{T} P_{2} \mathbf{S K} F_{\mathbf{w}}\right] J_{t}=\theta_{1} \mathbf{K}^{T} \mathbf{S}^{T} P_{1} I_{t}^{1}+\theta_{2} F_{\mathbf{w}}^{T} \mathbf{K}^{T} \mathbf{S}^{T} P_{2} I_{t}^{2}
$$

where $\left(J_{t}\right)_{x}$ and $\left(J_{t}\right)_{y}$ are the $x$ - and $y$-derivative matrices of the HR image $J_{t} .{ }^{T}$ is the transpose operator of the corresponding matrix; for example, $\mathbf{S}^{T}$ is the transpose of the downsampling $\mathbf{S}$. To simplify the equations, we use the following notation:

$$
P_{1}=\operatorname{diag}\left(\phi^{\prime}\left(\left\|\mathbf{S K} J_{t}-I_{t}^{1}\right\|^{2}\right)\right), P_{s}=\operatorname{diag}\left(\phi^{\prime}\left(\left\|\nabla J_{t}\right\|^{2}\right)\right), P_{2}=\operatorname{diag}\left(\phi^{\prime}\left(\left\|\mathbf{S K} F_{\mathbf{w}} J_{t}-I_{t}^{2}\right\|^{2}\right)\right)
$$

Here, diag denotes the diagonal weight matrices. The IRLS iterates between solving Eq. 3 and Eq. 4 are based on the current estimation to obtain the final ideal HR particle image $J_{t}$.

The solution for solving Eq. 2 is based on the assumption that the flow field $\mathbf{w}$, the blur kernel $\mathbf{K}$ and the noise levels $\theta_{1}$ and $\theta_{2}$ are given. The flow field $\mathbf{w}$ can be estimated with an optical flow Drulea and Nedevschi (2011). Hence, the unknown parameters in the generative models include $\mathbf{K}, \theta_{1}$ and $\theta_{2}$.

We use Bayesian maximum a posteriori estimation (MAP) to find the optimal solution:

$$
\underset{I, \mathbf{w},\left\{\theta_{1}, \theta_{2}\right\}}{\arg \max } p\left(J_{t}, \mathbf{w}, \mathbf{K},\left\{\theta_{1}, \theta_{2}\right\} \mid\left\{I_{t}^{1}, I_{t}^{2}\right\}\right)
$$

In addition, the sparsity on derivative filter responses is used to model the prior of HR image $J_{t}$, flow field w, and the blur kernel $K$ :

$$
p\left(J_{t}\right)=\frac{1}{Z_{J_{t}}(\eta)} \exp \left\{-\eta\left\|\nabla J_{t}\right\|\right\}, p(\mathbf{w})=\frac{1}{Z_{\mathbf{w}}(\lambda)} \exp \left\{-\lambda(\|\nabla u\|+\|\nabla v\|\}, p(\mathbf{K})=\frac{1}{Z_{\mathbf{K}}(\xi)} \exp \{-\xi\|\nabla \mathbf{K}\|\}\right.
$$

where $Z_{J_{t}}(\eta), Z_{\mathbf{w}}(\lambda)$ and $Z_{\mathbf{K}}(\xi)$ are normalization constants only dependent on parameters $\eta$, $\lambda$ and $\xi$, respectively. $u$ and $v$ are the horizontal and vertical components of the flow velocity $\mathbf{w}$.

Then, similar to Eq.2, when solving the noise levels $\theta_{1}$ and $\theta_{2}$, the HR image $J_{t}$ and the blur kernel $\mathbf{K}$ are given, and the Bayesian MAP estimation for the noise parameters has the following closed-form solution:

$$
\theta_{i}=\frac{\alpha+N_{q}-1}{\beta+N \bar{I}}, \quad \bar{I}=\frac{1}{N} \sum_{q=1}^{N}\left\|\left(I_{t}^{i}-\mathbf{S K} F_{\mathbf{w}_{i}} I\right)(q)\right\|, \quad i=1,2
$$

where $\bar{I}$ is the sufficient statistics, $N$ is the pixels of the LR image and $\alpha$ and $\beta$ are two constant parameters. For the blur kernel $\mathbf{K}$, the solution is similar to the HR image $J_{t}$; the upsampling $J_{t}$ and downsampling matrix $\mathbf{S}$ are given. The minimization formulation is as follows:

$$
\underset{\mathbf{K}}{\arg \min } \theta_{0}\left\|\mathbf{S} J_{t} \mathbf{K}-I_{t}^{1}\right\|+\eta\|\nabla \mathbf{K}\|
$$

which is also optimized by IRLS and not discussed in detail here.

With the iteration of the estimations of the noise controlling parameters $\theta_{1}^{m}$ and $\theta_{2}^{m}$, optical flow field $\mathbf{w}^{m}$, intermediate upsampling image $J_{t}^{m}$ and blur kernel $\mathbf{K}^{m}$, where $m$ is the current iteration number, the optimal HR particle image $J_{t}$ can be generated. We use 5 iterations to generate the HR particle image with $\eta=0.02$ for the image derivative, $\lambda=1,000$ for the optical flow field, $\xi=0.7$ for the derivative of the blur kernel and $\alpha=1$ and $\beta=0.1$ for noise. The upsampling scale is set to be 4 . 


\section{Experimental evaluations}

In this section, we illustrate the performance of the proposed SR reconstruction technology on synthetic and experimental images. First, we focus on the performance of the proposed BMFSR method and verify the advantage on a synthetic vortex flow in Sect. 3.1. Subsequently, in Sect. 3.2, real experimental microPIV data are tested to demonstrate the practicality of the novel SR reconstruction technology combining the multi-frame imaging system and the BMFSR algorithm, which utilizes the multi-frame displacement information.

Hereafter, we follow a standard method to quantitatively evaluate the experimental results by computing the peak signal-to-noise ratio (PSNR), the root mean square error (RMSE) and the average angle error (AAE) over $N$ pixels of the image:

$$
P S N R=10 \cdot \log _{10} \frac{255^{2}}{\frac{1}{N} \sum_{n=1}^{N}\left|J_{t}(n)-J(n)\right|^{2}}, R M S E=\sqrt{\frac{1}{N} \sum_{n=1}^{N}\left|\mathbf{w}_{n}^{e s}-\mathbf{w}_{n}^{t r}\right|^{2}}, A A E=\frac{1}{N} \sum_{n=1}^{N} \arccos \left(\frac{\mathbf{w}_{n}^{e s} \cdot \mathbf{w}_{n}^{t r}}{\left|\mathbf{w}_{n}^{e s}\right| \cdot\left|\mathbf{w}_{n}^{t r \mid}\right|}\right)
$$

where $J$ and $J_{t}$ are the ground truth and the reconstructed HR particle images, respectively. $\mathbf{w}^{t r}$ and $\mathbf{w}^{e s}$ denote the ground truth and the estimated velocity fields.

\subsection{Test on synthetic vortex flow}

First, we investigate the performance of the proposed BMFSR method on a synthetic vortex flow. Two pairs of HR particle images with $512 \times 512$ pixels are generated, whereby 7,864 particles with a mean diameter of 6 pixels and size variation of 0.3 pixels are randomly distributed. No out-of-plane motion or noise is considered, and the sheet thickness is set to 0.333. The displacement between these two pairs is set to 0.25 pixels and considered to be the background HR particle images of sensor 1 and sensor 2 . In this experiment, a synthetic velocity field is a Lamb-Ossen Vortex, similar to (Carlier and Wieneke, 2005). The exact solution of the Navier-Stokes equation is:

$$
u(r, \theta)=\frac{\Gamma_{0}}{2 \pi r}\left(1-e^{\frac{-r^{2}}{r_{0}^{2}}}\right)
$$

where $\Gamma_{0}$ is the initial circulation and $r_{0}$ is the core radius. In this study, $\Gamma_{0}=2000, r_{0}=50$ pixels. LR particle images are generated by downsampling the HR particle images above with a downsampling scale of 4. During dowmsampling, blur and noise are added to simulate the real scene.

The HR particle images of the background reconstructed by the traditional bicubic interpolation and reconstructed by the proposed BMFSR are shown in Fig. 2. As shown, the image reconstructed by BMFSR is more approximate to the background particle image. In addition, we compare the reconstruction quality with the evaluation indices PSNR, RMSE and AAE, and the results are shown in Fig.3. Compared to bicubic interpolation, the PSNR of our proposed method BMFSR is the highest, whether for $I_{t}^{1}$ or $I_{t}^{2}$. In Table 2, Bicubic-DCC denotes that the DCC results are set to be the background truth (Ours-DCC is the same) because both bicubic interpolation and our method estimate the velocity with an open source DCC and it has its inherent estimation error. The bold denotes the best and the underlined denotes the second here. In Table 2, it can be seen that the proposed method BMFSR performs better than the traditional bicubic interpolation method relative to whether the ground truth or the result of the basic DCC algorithm.

We present the energy maps and velocity distributions of different methods in Fig. 4. The comparison result suggests that the proposed BMFSR method outperforms the traditional bicubic interpolation method. In particular, at the bottom right, the bicubic result is worse, which may be caused by the blur and noise of the downsampling operator in generating LR particle images. However, the proposed BMFSR method can decrease the influence of blur and noise to some extent.

It can be concluded that the proposed BMFSR reconstruction algorithm is well adapted to SR reconstruction and shows great advantages in particle image reconstruction and HR velocity estimation compared to the traditional bicubic interpolation method. Then, the overall performance of the proposed super-resolution reconstruction technology is verified in a real experiment. 


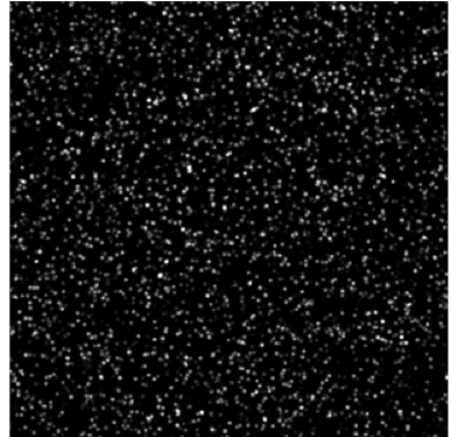

(a)

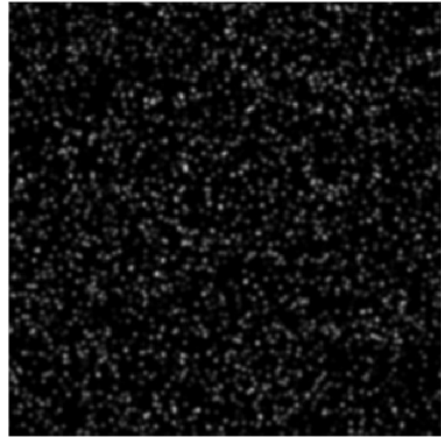

(b)

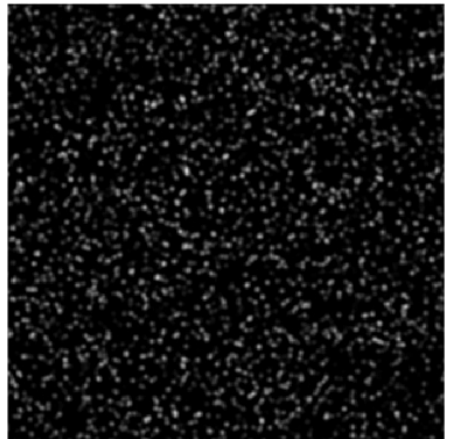

(c)

Figure 2: HR particle images of (a) the ground truth; (b) the traditional bicubic method; (c) the proposed BMFSR method.

Table 1. Particle image PSNRs

\begin{tabular}{|c|c|c|}
\hline Error Method & Bicubic & Ours \\
\hline PSNR of $I_{t}^{1}$ & 24.727 & $\mathbf{2 6 . 7 4 3}$ \\
\hline PSNR of $I_{t+\Delta t}^{1}$ & 24.730 & $\mathbf{2 6 . 7 9 8}$ \\
\hline
\end{tabular}

Table 2. Velocity errors of different methods

\begin{tabular}{|c|c|c|c|c|c|}
\hline Error Method & DCC & Bicubic & Ours & Bicubic-DCC & Ours-DCC \\
\hline RMSE & $\mathbf{0 . 0 6 8 0}$ & 0.1301 & $\underline{0.1013}$ & 0.0679 & 0.0503 \\
\hline AAE & $\mathbf{1 . 0 6 9 5}$ & 1.9231 & $\underline{1.5336}$ & 1.5783 & 0.9498 \\
\hline
\end{tabular}

Figure 3: Results of different methods on the reconstructed particle image PSNRs and the estimated velocity errors.

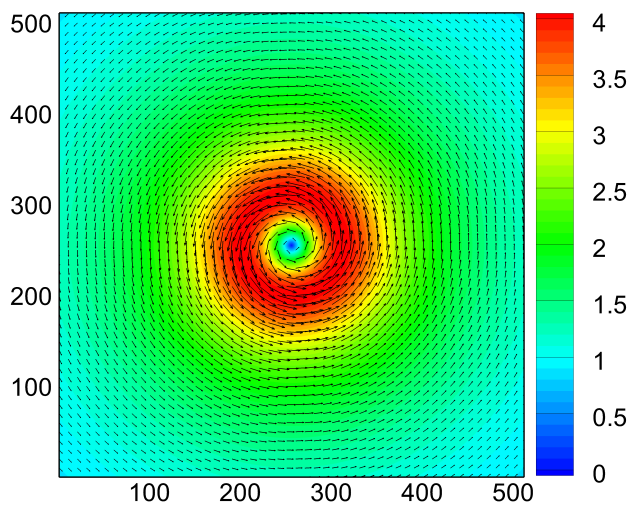

(a)

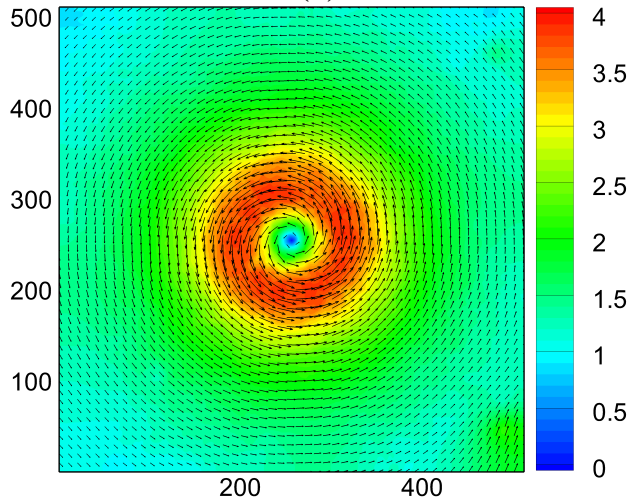

(c)

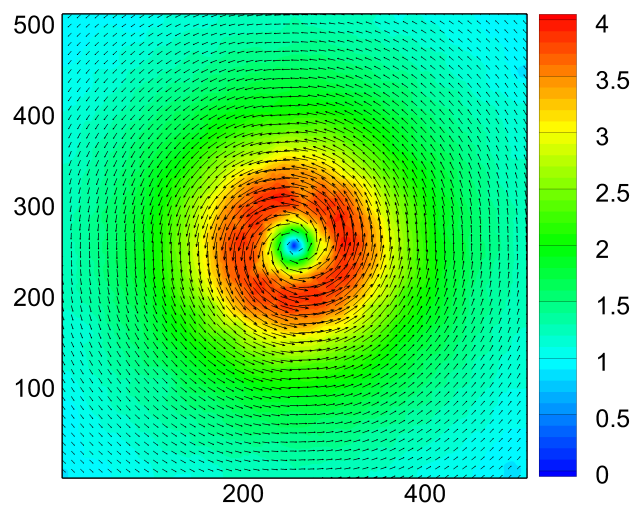

(b)

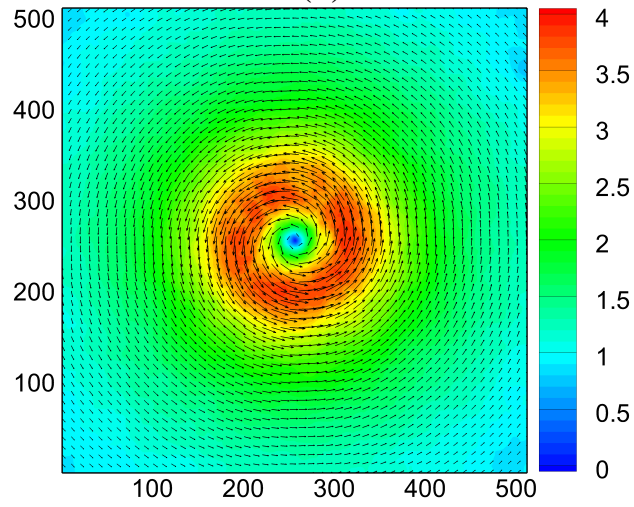

(d)

Figure 4: Energy maps and velocity distributions of different methods: (a) the ground truth; (b) result of DCC; (c) result of the traditional bicubic method; (d) result of the proposed BMFSR method. 


\subsection{Test on experimental laminar boundary layer flow}

To illustrate the generalization of the proposed method in the real flow field, a micro-PIV experiment was carried out to acquire the particle images of the laminar boundary layer with a constant inflow velocity, and the platform is shown in Fig. 5(a). Orange fluorescent particles with a diameter of $0.3 \mu \mathrm{m}$ were dispersed in distilled water and stored in a syringe. Then, an Era NE-1000 injection pump drove the syringe at 79 $\mu \mathrm{m} / \mathrm{s}$. The flow field is in a microfluidic chip, whose cross-section is $30 \mu \mathrm{m}$ wide $\times 28 \mu \mathrm{m}$ deep. The whole flow field was illuminated using fluorescent light (Nikon IntensiLight C-HGFI) with a wavelength of $532 \mathrm{~nm}$, which was the same as the light passed by the filter in the multi-frame imaging system and imaged by a Nikon Eclipse Ti-S inverted fluorescent microscope with a $60 \times$ magnification lens. The field is captured using the multi-frame imaging system with an interest region of $1,296 \times 408$ pixels. The flow field that we set is a laminar flow field, which has minimum velocity at the edge of the pipe and maximum velocity in the center of the pipe. In the experiment, we compare the performance of the traditional bicubic interpolation reconstruction method and our proposed BMFSR method. Note that the particle image that is used to generate the HR image of the bicubic interpolation method is only $I_{t}^{1}$; however, those of our proposed BMFSR are $I_{t}^{1}$ and $I_{t}^{2}$.
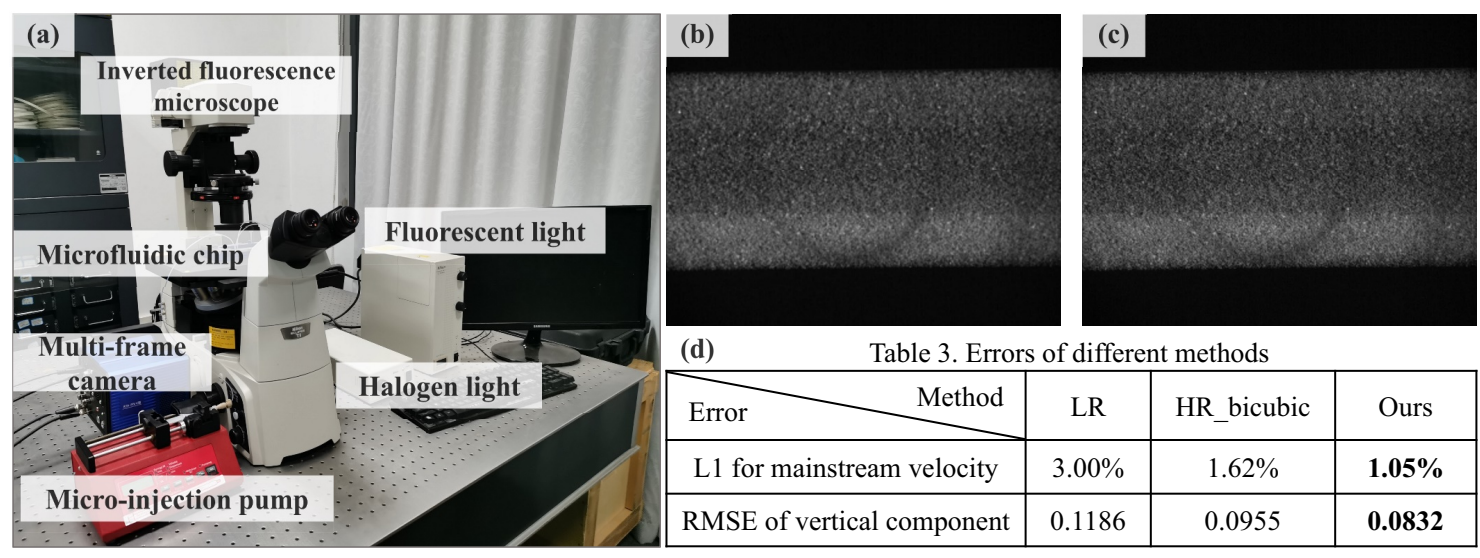

\begin{tabular}{|c|c|c|c|}
\hline Error $\quad$ Method & LR & HR_bicubic & Ours \\
\hline L1 for mainstream velocity & $3.00 \%$ & $1.62 \%$ & $1.05 \%$ \\
\hline RMSE of vertical component & 0.1186 & 0.0955 & 0.0832 \\
\hline
\end{tabular}

Figure 5: (a): Micro-PIV platform; (b) HR image with bicubic interpolation; (c) HR image with our proposed method; (d) errors of different methods.
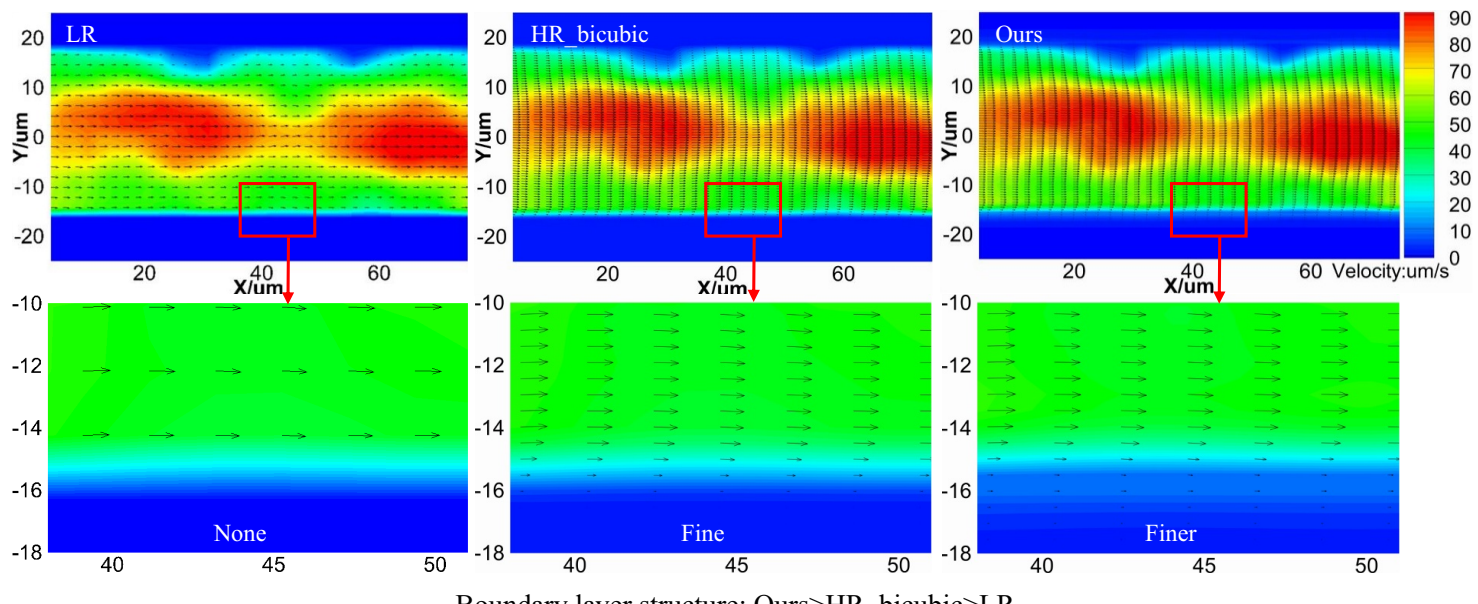

Boundary layer structure: Ours $>$ HR_bicubic $>$ LR

Figure 6: Top: the energy maps and velocity distributions of unsteady micro-PIV flow. Down: a close up in the region $-20 \leq \mathrm{Y} \leq-18$ pixels and $38 \leq \mathrm{X} \leq 51$ pixels.

Low-resolution images are directly acquired by the two sensors mentioned above. The HR particle images reconstructed from the traditional bicubic interpolation and our proposed method are shown in Fig.5(b) 
and (c), respectively. In comparison, the HR particle image reconstructed with our method is clearer because of the blur kernel estimation, and the result is consistent with the synthetic experiment above. The mainstream velocities measured are approximately $76.63 \mu \mathrm{m} / \mathrm{s}, 80.28 \mu \mathrm{m} / \mathrm{s}$ and $79.83 \mu \mathrm{m} / \mathrm{s}$ for $\boldsymbol{u}^{L R}, \boldsymbol{u}_{b i}^{H R}$ and $\boldsymbol{u}_{b a}^{H R}$, respectively; that is, the errors with the theoretical velocity are $3.00 \%, 1.62 \%$ and $1.05 \%$, as shown in Fig.5(d). Additionally, as shown in Fig.5(d), the proposed method obtains the lowest RMSE of the vertical component, which is 0.0832 . To further verify the performance of our method, we compare the energy maps and velocity distributions of three different methods: LR $\boldsymbol{u}^{L R}$, HR with bicubic reconstruction $\boldsymbol{u}_{b i}^{H R}$ and ours $\boldsymbol{u}_{b a}^{H R}$ in Fig. 6-top. Fig. 6-down shows the close up of these three velocity distributions in the region $-20 \leq \mathrm{Y} \leq-18$ pixels and $38 \leq \mathrm{X} \leq 51$ pixels. The upsampling scale factor of reconstruction here is 4 . The sampling rate in the horizontal direction of $\boldsymbol{u}_{b i}^{H R}$ and $\boldsymbol{u}_{b a}^{H R}$ is 4 . We can observe that the distribution of $\boldsymbol{u}_{b a}^{H R}$ is finer at the boundary. Therefore, it can be concluded that the proposed technology performs better in SR reconstruction.

\section{Conclusion}

In this paper, we propose a novel super-resolution (SR) reconstruction technology for particle image velocimetry from the perspective of SR particle image reconstruction using multi-frame displacement information. This technology consists of two parts: a multi-frame imaging system and a Bayesian-based multiframe SR (BMFSR) reconstruction algorithm. First, two correlated particle images are collected by the multi-frame imaging system at the same time with a constant displacement. Subsequently, these two images are utilized to generate an HR image with the proposed BMFSR method. This proposed SR reconstruction technology has been assessed by many experiments. First, a synthetic vortex flow is used as the benchmark to evaluate the performance of the proposed BMFSR algorithm. The results show that the proposed method can provide accurate reconstruction results. Compared to the traditional bicubic interpolation method, it also shows a great advantage in terms of the quality of the reconstructed particle image or the estimated highresolution (HR) velocity. The overall performance of the proposed SR reconstruction technology is verified with an experimental laminar boundary layer flow. The results show that the L1 error for the mainstream velocity and the RMSE of the vertical component of ours are both the lowest. In addition, the estimated flow structure of the laminar boundary layer of our proposed technology is also the finest.

\section{Acknowledgements}

This work was supported by the National Natural Science Foundation of China (grant no. 51875228) and the National Key R\&D Program of China (grant no. 2020YFA0405700).

\section{References}

Alfonsi G, Lauria A, and Primavera L (2013) Proper orthogonal flow modes in the viscous-fluid wavediffraction case. Journal of Flow Visualization and Image Processing 20:227-241

Brunton SL, Noack BR, and Koumoutsakos P (2020) Machine learning for fluid mechanics. Annual Review of Fluid Mechanics 52:477-508

Carlier J and Wieneke B (2005) Report 1 on production and diffusion of fluid mechanics images and data. fluid project deliverable 1.2. european project "fluid image analysis and description" (fluid). http://www.fluid.irisa.fr

Deng Z, He C, Liu Y, and Kim KC (2019) Super-resolution reconstruction of turbulent velocity fields using a generative adversarial network-based artificial intelligence framework. Physics of Fluids 31:125111

Drulea M and Nedevschi S (2011) Total variation regularization of local-global optical flow. in Proceedings of the 14th International IEEE Conference on Intelligent Transportation Systems, Washington, DC, USA, October 5-7

Dwivedi V, Parashar N, and Srinivasan B (2021) Distributed learning machines for solving forward and inverse problems in partial differential equations. Neurocomputing 420:299-316 
Fukami K, Fukagata K, and Taira K (2019a) Super-resolution analysis with machine learning for lowresolution flow data. in 11th International Symposium on Turbulence and Shear Flow Phenomena, Southampton, United Kingdom, July 30-August 2

Fukami K, Fukagata K, and Taira K (2019b) Super-resolution reconstruction of turbulent flows with machine learning. Journal of Fluid Mechanics 870:106-120

Gao H, Sun L, and Wang JX (2020) Super-resolution and denoising of fluid flow using physics-informed convolutional neural networks without high-resolution labels. arXiv preprint page arXiv:2011.02364

Gunes H and Rist U (2007) Spatial resolution enhancement/smoothing of stereo-particle-image-velocimetry data using proper-orthogonal-decomposition-based and kriging interpolation methods. Physics of Fluids 19:064101

He C and Liu Y (2017) Proper orthogonal decomposition-based spatial refinement of tr-piv realizations using high-resolution non-tr-piv measurements. Experiments in Fluids 58:1-22

Keys R (1981) Cubic convolution interpolation for digital image processing. IEEE Transactions on Acoustics, Speech, and Signal Processing 29:1153-1160

Liu B, Tang J, Huang H, and Lu XY (2020) Deep learning methods for super-resolution reconstruction of turbulent flows. Physics of Fluids 32:025105

Liu C and Sun D (2014) On bayesian adaptive video super resolution. IEEE Transactions on Pattern Analysis and Machine Intelligence 36:346-360

Liu C et al. (2009) Beyond pixels: exploring new representations and applications for motion analysis. Ph.D. thesis. Massachusetts Institute of Technology

Raissi M, Perdikaris P, and Karniadakis GE (2019) Physics-informed neural networks: A deep learning framework for solving forward and inverse problems involving nonlinear partial differential equations. Journal of Computational Physics 378:686-707

Seong JH, Song MS, Nunez D, Manera A, and Kim ES (2019) Velocity refinement of piv using global optical flow. Experiments in Fluids 60:1-13

Takehara K, Adrian R, Etoh G, and Christensen K (2000) A kalman tracker for super-resolution piv. Experiments in Fluids 29:S034-S041

Vejrazka J (2021) Pivsuite. https://www.mathworks.com/matlabcentral/fileexchange/45028-pivsuite 